\title{
Epidemiology of multidrug-resistant tuberculosis (MDR-TB) in Ethiopia: a systematic review and meta-analysis of the prevalence, determinants and treatment outcome
}

\author{
Tadele Girum ${ }^{1 *}$, Ebrahim Muktar ${ }^{1}$, Kifle Lentiro ${ }^{1}$, Habtamu Wondiye ${ }^{2}$ and Misgun Shewangizaw ${ }^{3}$
}

\begin{abstract}
Introduction: The emergence of MDR-TB remained a major public health threat particularly in developing countries. With increased prevalence and complexity of treatment, the burden of MDR-TB challenged the country. It is of an important; the epidemiology of drug resistant TB is not well understood. There are few studies conducted to assess the prevalence, determinants and treatment outcome of MDR-TB with inconclusive finding. Therefore, we aimed to conduct a systematic review and meta-analysis on Epidemiology of MDR-TB in Ethiopia, So that policy makers and other stalk holders could have pooled evidence on the problem to make a decision.

Methods: The review was conducted through a systematic literature search of articles published between 1997 and 2017. Five bibliographic databases and libraries: PubMed/Medline, Global Health Database, Embase, the Cochrane Library, and African Index Medicus were used. After cleaning and sorting, analysis was performed using STATA version 11. The pooled rate of MDR-TB prevalence, determinants and treatment outcome was estimated with a random-effects model. Heterogeneity was assessed by the $\mathrm{I}^{2}$ and publication bias through funnel plot.

Results: The 34 studies that were retained for final analysis enrolled a total of 7461 TB or MDR-TB patients. We found that 2.18\% (95\% Cl 1.44-2.92\%) of newly diagnosed and 21.07\% (95\% Cl 11.47-30.67\%) of previously treated patients have MDR-TB with overall prevalence of $7.24 \%(95 \% \mathrm{Cl} 6.11-8.37)$. History of previous treatment is the major determinant (pooled $\mathrm{OR}=4.78(95 \% \mathrm{Cl} 3.16-6.39)$ ), while contact history and adherence also contributed. In this review the pooled death computed among 5 articles showed that $12.25 \%$ (95\% Cl 9.39-15.11\%) of MDR-TB patients were died in the course of treatment. Complication, drug side effects and HIV infection were the main determinants for the death.

Conclusion and recommendation: The prevalence is by far higher than the previous reports. It is mainly associated with history of previous treatment along with contact history. However, the treatment outcomes are comparable with previous studies, yet it is a concern. Comorbidities, drug side effects and HIV sero-positivity were the determinants. Thus, proper treatment of drug susceptible TB and early detection and treatment of MDR-TB before complication develops along with prevention of drug side effect and contacts with MDR-TB cases are very important.
\end{abstract}

Keywords: MDR-TB, Tuberculosis, Drug resistance, Treatment outcome

\footnotetext{
* Correspondence: girumtadele@yahoo.com

${ }^{1}$ Department of Public health, college of Medicine and Health Sciences,

Wolkite University, Wolkite City, Ethiopia

Full list of author information is available at the end of the article
}

(c) The Author(s). 2018 Open Access This article is distributed under the terms of the Creative Commons Attribution 4.0 International License (http://creativecommons.org/licenses/by/4.0/), which permits unrestricted use, distribution, and reproduction in any medium, provided you give appropriate credit to the original author(s) and the source, provide a link to the Creative Commons license, and indicate if changes were made. The Creative Commons Public Domain Dedication waiver (http://creativecommons.org/publicdomain/zero/1.0/) applies to the data made available in this article, unless otherwise stated. 


\section{Background}

Multidrug-resistant tuberculosis (MDR-TB) is a type of TB that is resistant to the two most effective first-line drugs; Rifampicin and Isoniazid. It results from primary infection or may develop in the course of a patient's treatment [1]. The occurrence of MDR-TB is mainly attributable to human errors that predispose for resistance development, although genetic factors are believed to contribute to certain extent [2, 3]. The principal patient-related factor that predispose to MDR-TB is non-adherence to drug susceptible TB treatment [4].

Globally, $4.1 \%$ of new cases and $19 \%$ of previously treated TB cases were estimated to have had multidrug-resistant TB in 2016. In the same year, there were an estimated 600,000 incident cases of MDR/ RR-TB with cases of MDR-TB accounting for $82 \%$ of the total and there were an estimated 350,000 MDR/RR-TB cases among notified TB patients. However only 153,119 cases were confirmed which is much lower than the estimated cases because only $41 \%$ of TB cases have been tested for resistance. There were about 240,000 deaths from MDR/RR-TB in 2016 [5].

In 2016, the 30 high MDR-TB burden countries accounted for $89.7 \%$ of the global incident cases of MDR-TB. While India, China and the Russian Federation alone accounted $47 \%$ of the global burden. In Africa, where little data is available, an estimated 93,000 cases were emerged in 2016 with estimated incidence of $2.7 \%$ among new and $14 \%$ among previously treated cases, but the vast majority of them went un-diagnosed. Ethiopia is one of the 30 MDR-TB high-burden countries, with an estimated 5800 cases reported in 2016 with the prevalence rate of $2.7 \%$ among new cases and $14 \%$ among previously treated patients $[5,6]$.

Globally, 129,689 of the 153,119 notified cases of multidrug-resistant $\mathrm{TB}$ and rifampicin resistant $\mathrm{TB}$ (MDR/RR-TB) in 2016 were enrolled in treatment. It means $85 \%$ of diagnosed cases or only $22 \%$ of the estimated incident MDR/RR-TB cases initiated treatment by the year. Which shows improvement in coverage and access to MDR TB treatment; however the cure rate is low and on the other hand mortality, default and failure are high. Only 50\% of the MDR-TB patients in the 2012 cohort of detected cases were successfully treated, $16 \%$ died, and $10 \%$ failed [7]. Similarly, $54 \%$ of MDR/RR-TB cases in the 2014 cohort have successful outcome by 2016 [5].

The emergence of MDR-TB is a threat for the populations of resource-limited countries. Generally, the low socioeconomic status of the people, high prevalence of infectious diseases and limited access to well-equipped health care facilities worsens the effect of MDR-TB. Furthermore, poor treatment outcomes, longer treatment time (about two years), higher treatment costs, and many more complications make MDR-TB a more complex disease than TB $[8,9]$.

The overall epidemiology of drug resistant $\mathrm{TB}$ is not well understood in Ethiopia [10-12]. Accordingly there are few studies conducted to assess the prevalence, determinants and treatment outcome of MDR-TB with inconclusive findings. There are also other researches with finding of null impact of determinants on the prevalence and treatment outcome of MDR-TB. Therefore we aimed to conduct a systematic review on the epidemiology of MDR-TB in Ethiopia to assess the prevalence, determinants and treatment outcome of MDR-TB. So that policy makers and other stalk holders could have pooled evidence on the problem to make a decision.

\section{Methods}

\section{Literature search strategy}

This systematic reviews and meta analyses was conducted in accordance to the PRISMA (Preferred Reporting Items for Systematic Reviews and Meta-Analyses: guidance for reporting of systematic reviews and meta analyses) [13] through a systematic literature search of articles published between 1997 and 2017 containing information on MDR-TB prevalence, determinants and treatment outcome. Search engines, Electronic bibliographic databases and libraries: PubMed/Medline, Global Health Database, Embase, the Cochrane Library, African Index Medicus and Google scholar were used to retrieve articles published with in the study period. Combination of search terms: "Resistant TB", "MDRTB", "Isoniazid and rifampicin resistance", "HIV and TB", "Re-treatment and resistance", "TB treatment" and the names of MDR-TB treatment hospitals were used with (AND, OR, NOT) Boolean (Search) Operators. In addition the reference lists of primary and pertinent review articles were also uploaded into an EndNote XI library (EndNote, Carlsbad, CA, USA) to identify cited studies not captured by the electronic search and after all checked for duplications.

\section{Selection /eligibility criteria}

Studies that reported the prevalence of MDR-TB among new and/or previously treated patients, studies which compared two groups or reported determinants and studies which reported treatment outcome of MDR-TB that conducted in Ethiopia and published in English language regardless of the design and setting were used. Studies were also eligible for the review regardless of their study time. Studies conducted among presumptive MDR-TB cases only were excluded from the analysis to minimize over reporting. However studies conducted in MDR-TB referral centers which included both presumptive and all other cases were included. 


\section{Operational definition}

The following definition in accordance with the MDR-TB guide line $[5,14]$ were used in this meta-analysis

- Drug-resistant TB - TB that is resistant to any firstline anti-tuberculosis drug

- MDR-TB is TB that caused by strains of M.tuberculosis that are resistant to at least INH and RMP.

- MDR-TB among new cases (Primary drug resistance) is defined as resistance to isoniazid and rifampin drugs in patients that have never been treated for TB. This reflects person-to-person transmission of drug-resistant TB bacilli.

- MDRTB among previously treated TB patients (Acquired drug resistance) is defined as resistance to isoniazid and rifampin drugs in patients that have been treated for TB. This reflects drug resistance acquired during $\mathrm{TB}$ treatment but may also reflect infection or re- infection with resistant TB bacilli.

- Presumptive MDR-TB is as smear positive previously treated patients who define as relapse, return after default, and failure; new smear positive pulmonary TB patients whose sputum remains smear positive at month 2 or 3 of treatment; symptomatic close contacts of known MDR-TB patient, and new smear positive with Human Immunodeficiency Virus (HIV) infected patients.

- Medical complication: is a comorbid condition diagnosed as secondary diagnosis in tuberculosis patients

\section{Outcome of interest}

This review was intended to measure the prevalence of MDR-TB among newly and/or previously treated TB patients. MDR-TB prevalence was calculated for the total sample and stratified for new and re-treatment groups. The odds of possible determinants were calculated for two variables and for others it was summarized. Lastly treatment outcome was measured in terms of mortality, cure and default rate.

\section{Data extraction and abstraction}

The titles and abstracts derived through the primary electronic search were thoroughly assessed for possibility of reporting the intended outcome and filtered for potential eligibility. From each eligible research, the following information was extracted based on the preformed database (Excel, Microsoft, 2010) format: about author, characteristic of the study participants, studies (study design, cohort size, setting), prevalence of MDR-TB, Treatment outcome, year of publication, year of study start and end, eligibility criteria,, etc. All data were extracted independently and in duplicate using a standardized extraction form. Returned abstracts were reviewed and full texts retrieved if they contained relevant information. Mean way, each selected research was assessed for methodological quality and possibility of bias.

\section{Data analysis}

After cleaning and sorting the final database was exported into Stata 11.0 for analysis (Stata, College Station, TX, USA). An outcome of interest was rate of MDR-TB among different groups, rate of mortality and their determinants. Estimate of MDR-TB prevalence was assessed for each study and standardized mean with 95\% confidence interval was used. These were calculated with a random-effects model according to the DerSimonian and Laird method [15]. Heterogeneity was assessed by the $\mathrm{I}^{2}$ and values greater than $50 \%$ considered representing significant heterogeneity. When heterogeneity between studies was found to be significant, pooled estimates were based on random-effect models and the Hedges method of pooling. Results were displayed visually in forest plots. Bias was investigated by construction of funnel plots and Analysis was performed using the 'metan' and related functions in STATA version 11 (College Station, TX).

\section{Results}

\section{Studies included}

Our initial electronic database search with the chosen search terms identified 924 citations in the form of abstract, bibliography and full text research. Out of these, 427 researches which do not match with our objectives were excluded after reviewing their titles and abstracts. Then the remaining citations were transferred to endnote and cleaned for duplications and 98 articles were identified for full text review. Of the 98 articles reviewed in full text, 34 articles [16-49] were retained for final analysis based on the inclusion-exclusion criteria and quality assessment and 64 studies were removed prior to analysis for different reasons: 21 studies reported the prevalence of drug resistance other than MDR, 27 removed for lack of clear outcomes, 13 removed for reporting outcome among Non-resistant TB cases, 3 overlapped with larger studies. The study selection process is presented in (Fig. 1).

\section{Description of findings}

The 34 studies that were retained for final analysis enrolled a total of $7461 \mathrm{~TB}$ or MDR-TB patients [16-49]. With duplicates (repeated count), 23 cross-sectional studies measured prevalence of MDR-TB among 3477 New and /or previously treated patients [16-38], 14 cross-sectional and case control studies assessed determinants of MDR-TB among 3534 patients [24, 26, 28, $32,33,35,37-44]$ and the rest 5 cohort studies assessed the treatment outcome and its determinants among 


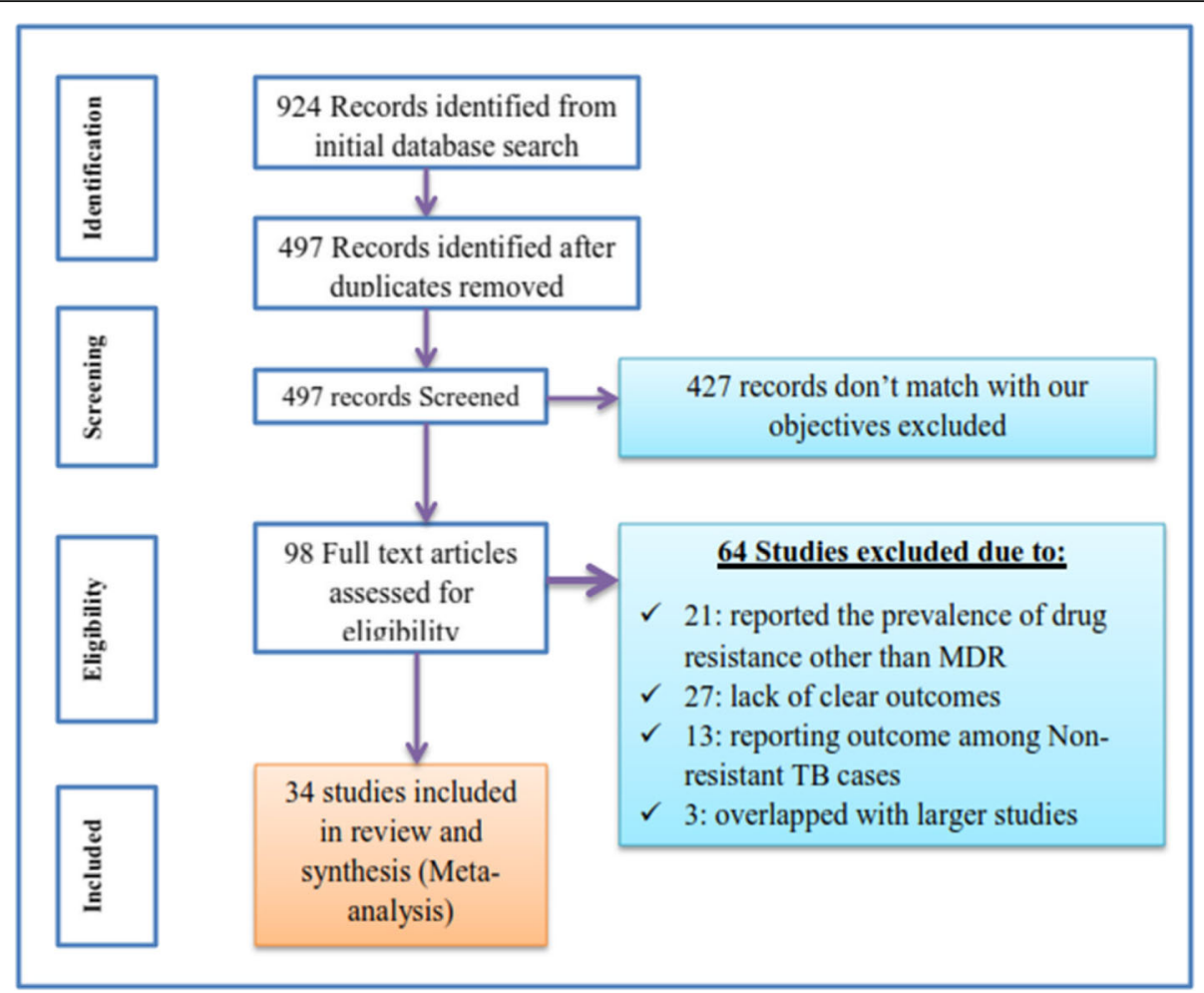

Fig. 1 Flow chart for study search, selection and screening for the review

1538 MDR-TB patients [45-49]. Except one, all the studies were institution based researches conducted in different regions of Ethiopia and published between 1997 and 2017 with English language. Thirteen studies were conducted in Addis Ababa, where two of the first MDR-TB treatment centers were located and the rest were conducted in other referral centers and TB treatment hospitals. Survey characteristics are described in (Table 1).

\section{Prevalence of MDR-TB}

Of the 23 researches which reported MDR-TB prevalence, 14 researches reported prevalence of MDR-TB among previously treated patients, 14 researches reported among New PTB patients, 20 reported any first line drug resistance, 17 measured prevalence of isoniazid (INH) resistance and 16 reported prevalence of Rifampicin resistance (Table 2).

Overall, the prevalence of MDR-TB ranged from 0.5 to $46.3 \%$, with a pooled prevalence of $7.24 \%$ (95\% CI $6.11-$ 8.37) (The pooled estimate was from random effect analysis). The proportion of MDR-TB among all TB cases varies from place to place. Among the reviewed researches, six articles reported high prevalence of MDR-TB in the range of 10.2-46.3\% [20-22, 26, 32, 38]; Three of them being reported in Addis Ababa [20-22]. The highest MDR-TB prevalence with a rate of $46.3 \%$ was reported by Abate et al. [22] in Addis Ababa among previously treated PTB patients and as high as a prevalence of $43 \%$ was also reported among New and Previously treated PTB patients by Agonafir et al. [21] (Fig. 2).

Studies conducted in other parts of the country also reported similar results. A study conducted in south western Ethiopia indicated that $31.4 \%$ of previously treated PTB patients have MDR-TB [32]. Additionally, $11.8 \%$ of MDR-TB was reported by Hussein et al. in 2013 [26] and $10.2 \%$ by Brhane et al. in 2017 [38] among all cases. On the other hand, three of these studies reported MDR-TB prevalence of below 1\% [17-19]. .

The pooled prevalence of MDR-TB among newly diagnosed TB cases was $2.18 \%$ (95\% CI 1.44-2.92\%) (Fig. 3). The highest prevalence among new cases was reported to be 11.8 by Hussien et al. [26]. The overall prevalence of MDR-TB among previously treated TB cases was $21.07 \%$ (95\% CI 11.47-30.67\%) (Fig. 4). The heterogeneity test indicated that there is statistical significant variation among pooled estimate of MDR-TB in previously treated cases and new cases. Therefore, the pooled estimate was from random effect analysis in both cases. The observed variation of MDR-TB prevalence among newly diagnosed and previously treated cases was statistically significant $(P<0.001)$.

In our review, 20 researches that enrolled a total of 3087 patients assessed the prevalence of any first line 
Table 1 Characteristics of studies included in the review

\begin{tabular}{|c|c|c|c|c|c|}
\hline Studies included in the review & Study area & Study population & Study design & Study period & No of patients \\
\hline [16] Demissie et al. 1997 & Addis Ababa & New PTB patients & Cross-sectional study & 1994 & 167 \\
\hline [17] Gebeyehu, 2001 & Arsi Zone & PTB patients & Cross-sectional study & 1997-98 & 195 \\
\hline [18] Bruchfeld, 2002 & Addis Ababa & PTB patients & Cross-sectional study & 2000 & 121 \\
\hline (19)Asmamaw et al., 2008 & Addis Ababa & New PTB patients & Cross-sectional study & $2004-5$ & 173 \\
\hline [20] Meskel et al. 2008 & Addis Ababa & Previously treated PTB & Cross-sectional study & $2001-2$ & 84 \\
\hline [21] Agonafir, 2010 & Addis Ababa & PTB patients & Cross-sectional study & $2005-6$ & 107 \\
\hline [22] Abate et al., 2012 & Addis Ababa & Previously treated PTB & Retrospective & $2004-8$ & 376 \\
\hline [23] Abebe et al. 2012 & South western Ethiopia & New PTB patients & Cross-sectional study & $2010-11$ & 136 \\
\hline [24] Tessema et al. 2012 & North Ethiopia & PTB patients & Cross-sectional study & 2009 & 260 \\
\hline [25] Yimer et al. 2012 & ARS major towns & New PTB patients & Cross-sectional study & 2008 & 93 \\
\hline [26] Hussein et al. 2013 & Bahirdar, Fitche \& Ambo & PTB patients & Cross-sectional study & 2011 & 102 \\
\hline [27] Biadglegne et al.2014 & Bahirdar, Gondar \& Dessie & TB lymphadenitis & Cross-sectional study & 2012 & 226 \\
\hline [28] Esmael et al. 2014 & Eastern ARS & PTB patients & Cross-sectional study & $2010-11$ & 230 \\
\hline [29] Daniel et al. 2014 & Debre Birhan & PTB patients & Cross-sectional study & 2013-14 & 40 \\
\hline [30] Seyoum et al. 2014 & Eastern Ethiopia & New PTB patients & Cross-sectional study & $2011-13$ & 357 \\
\hline [31] Tekle et al. 2014 & Benishangul \& Awi & PTB patients & Cross-sectional study & 2013-14 & 87 \\
\hline [32] Abdella et al., 2015 & South western Ethiopia & Previously treated PTB & cross sectional & $2012-13$ & 79 \\
\hline [33] Adane et al. 2015 & East Gojjam & PTB patients & Cross-sectional study & $2011-12$ & 89 \\
\hline [34] Maru et al. 2015 & Dessie & PTB patients & Cross-sectional study & $2012-13$ & 118 \\
\hline (35)Mekonnen et al. 2015 & North Gondar & PTB patients & Cross-sectional study & 2014 & 124 \\
\hline [36] Ali et al. 2016 & South Ethiopia & PTB patients & cross sectional & 2013 & 109 \\
\hline [37] Hamussie et al. 2016 & Arsi Zone & PTB patients & Cross-sectional study & 2013-14 & 106 \\
\hline [38] Brhane et al., 2017 & Jigjiga & PTB patients & cross sectional & NR & 98 \\
\hline [39] Mulisa et al. 2015 & Oromia Region & MDR \& Non MDR TB & Case control study & 2013-14 & 439 \\
\hline [40] Dessalegn et al. 2016 & Addis Ababa & MDR \& Non MDR TB & Case control study & NR & 206 \\
\hline [41] Hirpa et al. 2013 & Addis Ababa & MDR \& Non MDR TB & Case control study & $2011-12$ & 268 \\
\hline [42] Deressa et al. 2014 & Addis Ababa & MDR \& Non MDR TB & Case control study & 2013 & 384 \\
\hline [43] Assefa et al. 2017 & Addis Ababa & MDR \& Non MDR TB & Case control study & 2013 & 710 \\
\hline [44] Tadesse et al. 2015 & Addis Ababa & MDR \& Non MDR TB & cross sectional & $2008-11$ & 439 \\
\hline [45] Molalign et al. 2015 & Addis Ababa \& Gondar & MDR TB & Cohort & $2011-14$ & 342 \\
\hline [46] Alene et al. 2016 & North west Ethiopia & MDR TB & retrospective cohort & 2010-15 & 242 \\
\hline [47] Getachew et al. 2013 & Addis Ababa & MDR TB & retrospective cohort & $2011-12$ & 188 \\
\hline [48] Meressa et al. 2015 & Addis Ababa & MDR TB & Cohort & 2009-14 & 612 \\
\hline [49] Girum et al. 2017 & South Ethiopia & MDR TB & retrospective cohort & $2011-16$ & 154 \\
\hline
\end{tabular}

ARS- Amhara Regional state MDR- TB = Multidrug resistant tuberculosis

anti TB drug resistance and $58.6 \%$ are found to be resistant. Of the 17 researches that assessed the prevalence of INH resistance, $51.4 \%$ were found to have resistant TB to INH. Similarly, $32.9 \%$ of the 2460 patients have Rifampicin resistance (Table 2).

\section{Determinants of MDR-TB}

The reviewed articles indicated that previous exposure to TB treatment, contact history to known MDR-TB cases and previous treatment outcome were known be associated with prevalence of MDR-TB $[24,26,28,32,33,35,37-44]$. In this review, MDR-TB was found to be significantly associated with a previous history of anti-TB drug treatment. The risk of having MDR-TB was about 5 times higher in individuals with a previous history of anti-TB treatment. Except Hussien et al.'s report [26], all of the 12 researches reported that previous treatment is positively associated with MDR-TB with a pooled odds of 4.78 (95\% CI 3.16-6.39) (Fig. 5). 
Table 2 Prevalence of primary and multidrug resistance

\begin{tabular}{|c|c|c|c|c|c|c|c|c|c|}
\hline \multirow[t]{2}{*}{ Studies included } & \multicolumn{3}{|c|}{ Sample size } & \multicolumn{3}{|c|}{ Primary drug resistance \% } & \multicolumn{3}{|c|}{ MDR \% among } \\
\hline & New & Re-treated & $\overline{\text { Total }}$ & Any drug & $\mathrm{INH}$ & $\overline{\mathrm{RMP}}$ & New & re-treatment & over all \\
\hline Demissie et al. 1997 & 167 & 0 & 167 & 15.6 & 8.4 & 1.8 & $N R$ & NR & 1.2 \\
\hline Gebeyehu, 2001 & 195 & 19 & 195 & 19.5 & 7.6 & 0 & NR & NR & 0.5 \\
\hline Bruchfeld, 2002 & 103 & 18 & 121 & 14 & 8.3 & 2.5 & NR & $N R$ & 0.8 \\
\hline Asmamaw et al., 2008 & 173 & 0 & 173 & 21.4 & 13.3 & 1.2 & $N R$ & $N R$ & 0.6 \\
\hline Meskel et al. 2008 & 0 & 84 & 84 & 53.6 & NR & NR & $N R$ & 26.2 & 26.2 \\
\hline Agonafir, 2010 & 44 & 63 & 107 & 60.8 & 54.2 & 43.9 & 2.3 & 71.4 & 43 \\
\hline Abate et al., 2012 & 0 & 376 & 376 & 72.9 & 56.1 & 46.5 & & 46.3 & 46.3 \\
\hline Abebe et al. 2012 & 136 & 0 & 136 & 18.4 & 13.2 & 2.2 & 1.5 & $N R$ & 1.5 \\
\hline Tessema et al. 2012 & 214 & 46 & 260 & 15.8 & 13.8 & 5.8 & 3.7 & 10.9 & 5 \\
\hline Yimer et al. 2012 & 93 & 0 & 93 & 30.1 & 20.4 & 3.2 & 1.1 & $N R$ & 1.1 \\
\hline Hussein et al. 2013 & 93 & 9 & 102 & 36.3 & 29.4 & 13.7 & 11.8 & 11.1 & 11.8 \\
\hline Biadglegne et al.2014 & 213 & 13 & 226 & NR & $N R$ & $N R$ & 1.4 & 0 & 1.3 \\
\hline Esmael et al. 2014 & 165 & 65 & 230 & 33.5 & $N R$ & $N R$ & 1.8 & 18.5 & 6.5 \\
\hline Daniel et al. 2014 & $N R$ & NR & 40 & $N R$ & $N R$ & $N R$ & 6.3 & 12.5 & 7.5 \\
\hline Seyoum et al. 2014 & 357 & 0 & 357 & 23 & 14 & 2.8 & 1.1 & NR & 1.1 \\
\hline Tekle et al. 2014 & 75 & 12 & 87 & 16.5 & 12.6 & 2.3 & 1.3 & 8.3 & 2.3 \\
\hline Abdella et al., 2015 & 79 & 0 & 79 & 58.6 & 51.4 & 32.9 & $N R$ & 31.4 & 31.4 \\
\hline Adane et al. 2015 & 77 & 12 & 89 & 20.23 & 6.74 & 5.62 & 1.3 & 16.7 & 3.4 \\
\hline Maru et al. 2015 & 103 & 15 & 118 & 17.8 & 13.55 & 0 & 0 & 13.3 & 1.7 \\
\hline Mekonnen et al. 2015 & 88 & 36 & 124 & $N R$ & $N R$ & $N R$ & 2.3 & 13.9 & 5.6 \\
\hline Ali et al. 2016 & $N R$ & $N R$ & 109 & 24.77 & 7.34 & 5.5 & $N R$ & $N R$ & 3.67 \\
\hline Hamussie et al. 2016 & 85 & 21 & 106 & 21.7 & 13.2 & 7.5 & 2.4 & 14.3 & 4.7 \\
\hline Brhane et al., 2017 & 67 & 31 & 98 & 18.4 & $N R$ & 8.2 & 4.5 & 22.6 & 10.2 \\
\hline Weighted mean & - & - & - & 31.1 & 21.65 & 13.4 & $2.18^{*}$ & $21.07^{*}$ & $7.24^{*}$ \\
\hline Total N & 2527 & 820 & 3477 & 3087 & 2675 & 2460 & 2527 & 820 & 3477 \\
\hline
\end{tabular}

INH-Isoniazid, RMP-rifampicin, NR $=$ Not reported * value indicates polled prevalence from random effect model

However, in this review HIV was not significantly associated with MDR-TB prevalence. Of the 11 studies which reviewed to evaluate the association between MDR-TB and HIV only 3 researches by Tadesse et al. [44], Mulisa et al. [39] and Assefa et al. [43] reported significant positive association. In contrast to these, another 3 researches $[26,40,41]$ showed preventive association and 5 researches [24, 32, 33, 35, 38] sowed non-significant positive association. Over all pooled odds of HIV was 1.17 (95\% CI 0.43-1.91) which indicates a positive but insignificant association (Fig. 6).

In addition to these factors, exposure to a known MDR-TB case (Contact history) in Mulisa et al. [39], Assefa et al. [43] and Brhane et al. [38], treatment adherence and drug side effect in Hirpa et al. [41] previous treatment outcome in Meressa et al. [48] were found to have a positive association with MDR-TB in Ethiopia. However a pooled estimate was not computed due to the smaller number of studies which assessed each variable.

\section{Treatment outcome of MDR-TB}

Concerning the outcomes, this meta-analysis revealed that $8.4-13.9 \%$ of MDR-TB patient died during treatment and $25-64.7 \%$ were get cured, another $10-16.9 \%$ were completed treatment [45-49]. According to Molalign et al. [45] of the 342 MDR-TB patients $10.8 \%$ were died and the median survival time was 16 months. In another study $13 \%$ of patients were died and $54 \%$ were cured [46]. A research by Girum et al. [49] also reported that $8.4 \%$ of patients were died, $25.3 \%$ were cured and $16.9 \%$ completed their treatment. In this review the pooled death computed among 5 researches showed that 12.25\% (95\% CI 9.39-15.11\%) of MDR-TB patients were died (Fig. 7). Presence of medical complication, drug side effect, HIV sero-positivity and baseline weight were the most common reasons for the death [47, 49].

\section{Discussion}

This study assessed the prevalence of MDR-TB among newly diagnosed and previously treated patients, 


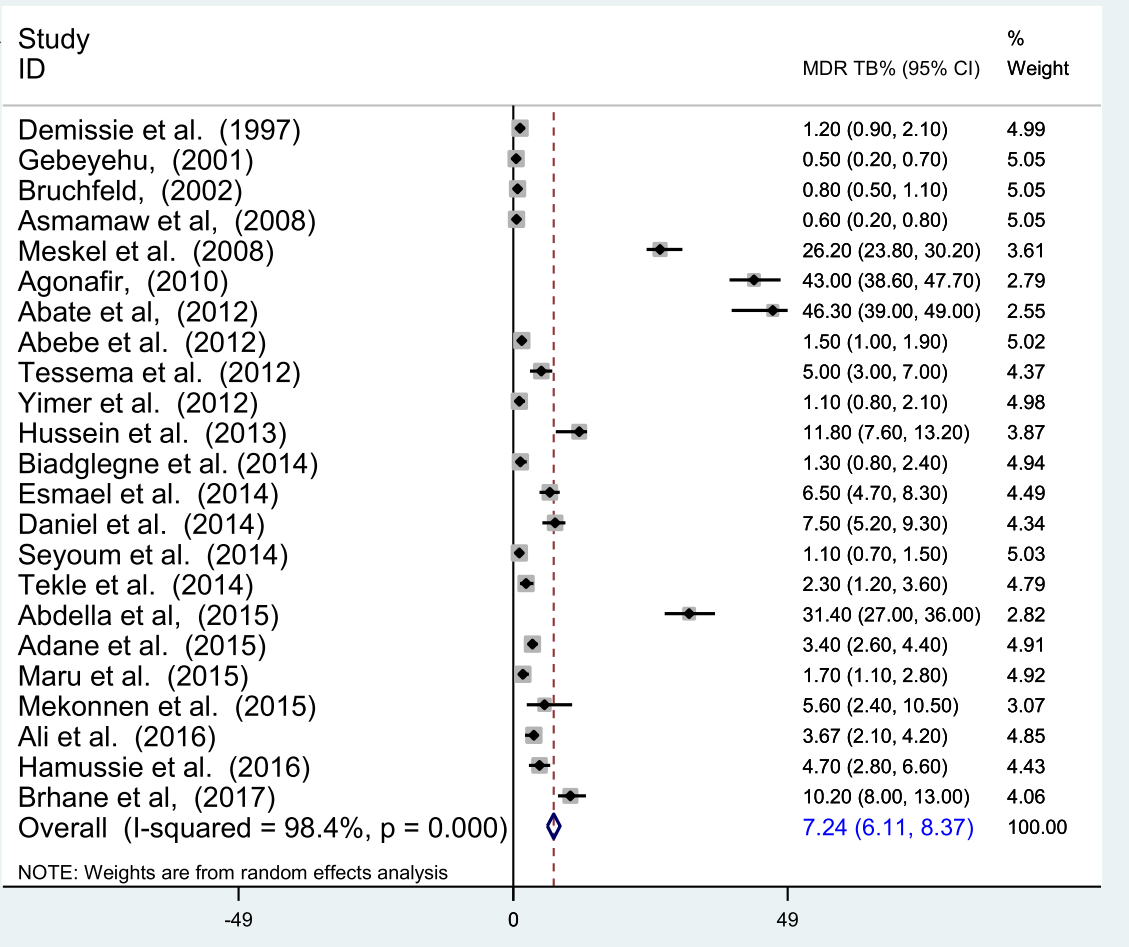

Fig. 2 Forest plot showing the prevalence of MDR-TB among the total samples

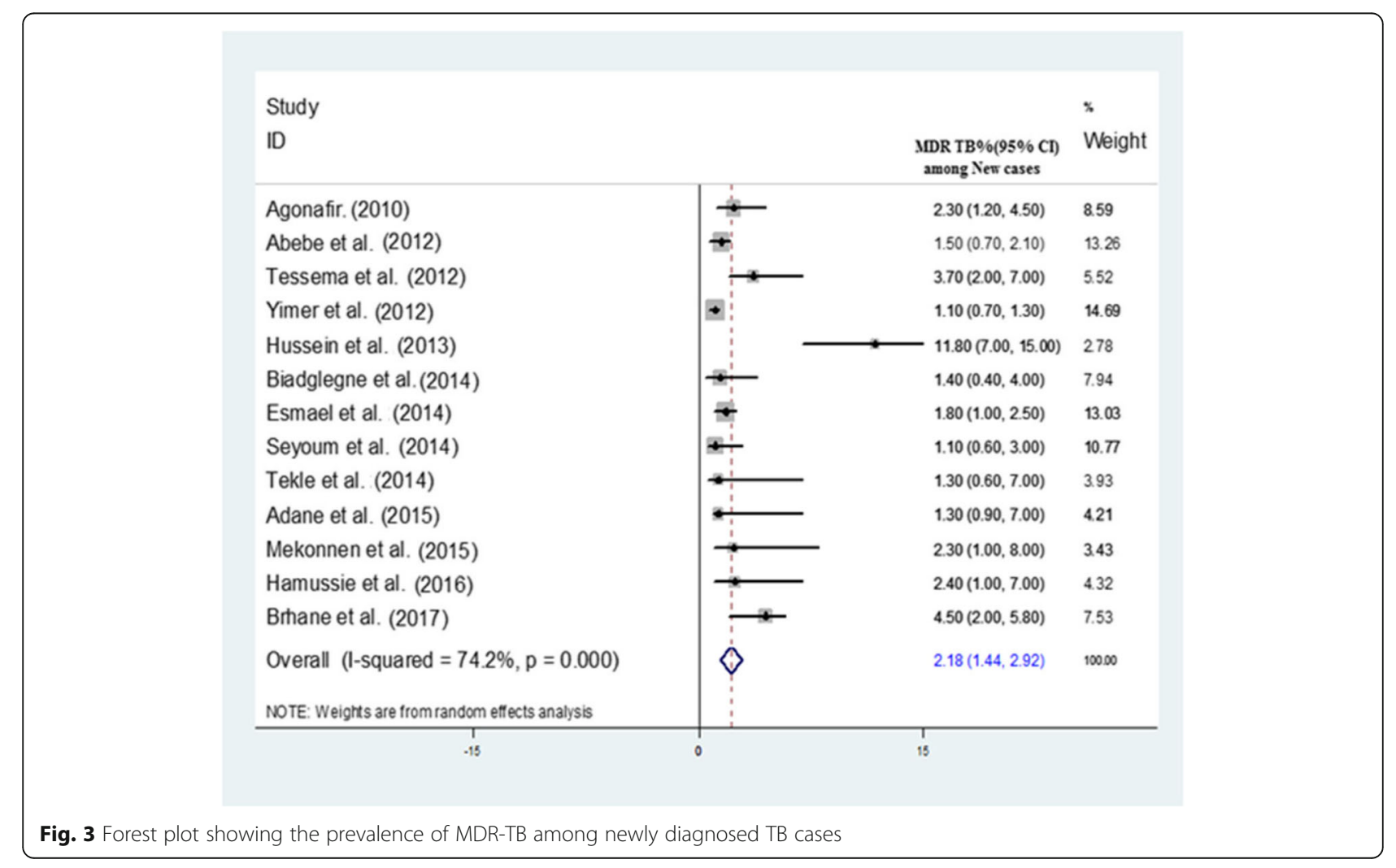


Study

ID

Meskel et al. (2008)

Agonafir, (2010)

Abate et al, (2012)

Tessema et al. (2012)

Hussein et al. (2013)

Biadglegne et al. (2014)

Esmael et al. (2014)

Daniel et al. (2014)

Tekle et al. (2014)

Abdella et al, (2015)

Adane et al. (2015)

Maru et al. (2015)

Mekonnen et al. (2015)

Hamussie et al. (2016)

Brhane et al, 2017 (2017)

Overall (I-squared $=97.0 \%, p=0.000)$

NOTE: Weights are from random effects analysis

$-82$
$\%$

MDR TB\% $(95 \% \mathrm{Cl}) \quad$ Weight

$26.20(18.00,36.00) \quad 6.72$

$\longrightarrow 71.40(63.00,82.00) \quad 6.68$

$46.30(32.00,53.00) \quad 6.58$

$10.90(5.00,23.00) \quad 6.72$

$11.10(6.00,23.00) \quad 6.77$

$0.00(0.00,1.00) \quad 7.15$

$18.50(11.00,30.00) \quad 6.68$

$12.50(5.00,28.00) \quad 6.48$

$8.30(2.00,24.00) \quad 6.53$

$31.40(23.00,46.00) \quad 6.48$

$16.70(9.00,26.00) \quad 6.77$

$13.30(7.00,17.00) \quad 7.01$

$13.90(6.00,29.00) \quad 6.48$

$14.30(5.00,35.00) \quad 6.09$

$22.60(16.00,31.00) \quad 6.85$

$21.07(11.47,30.67) \quad 100.00$

Fig. 4 Forest plot showing the prevalence of MDR-TB among previously treated cases

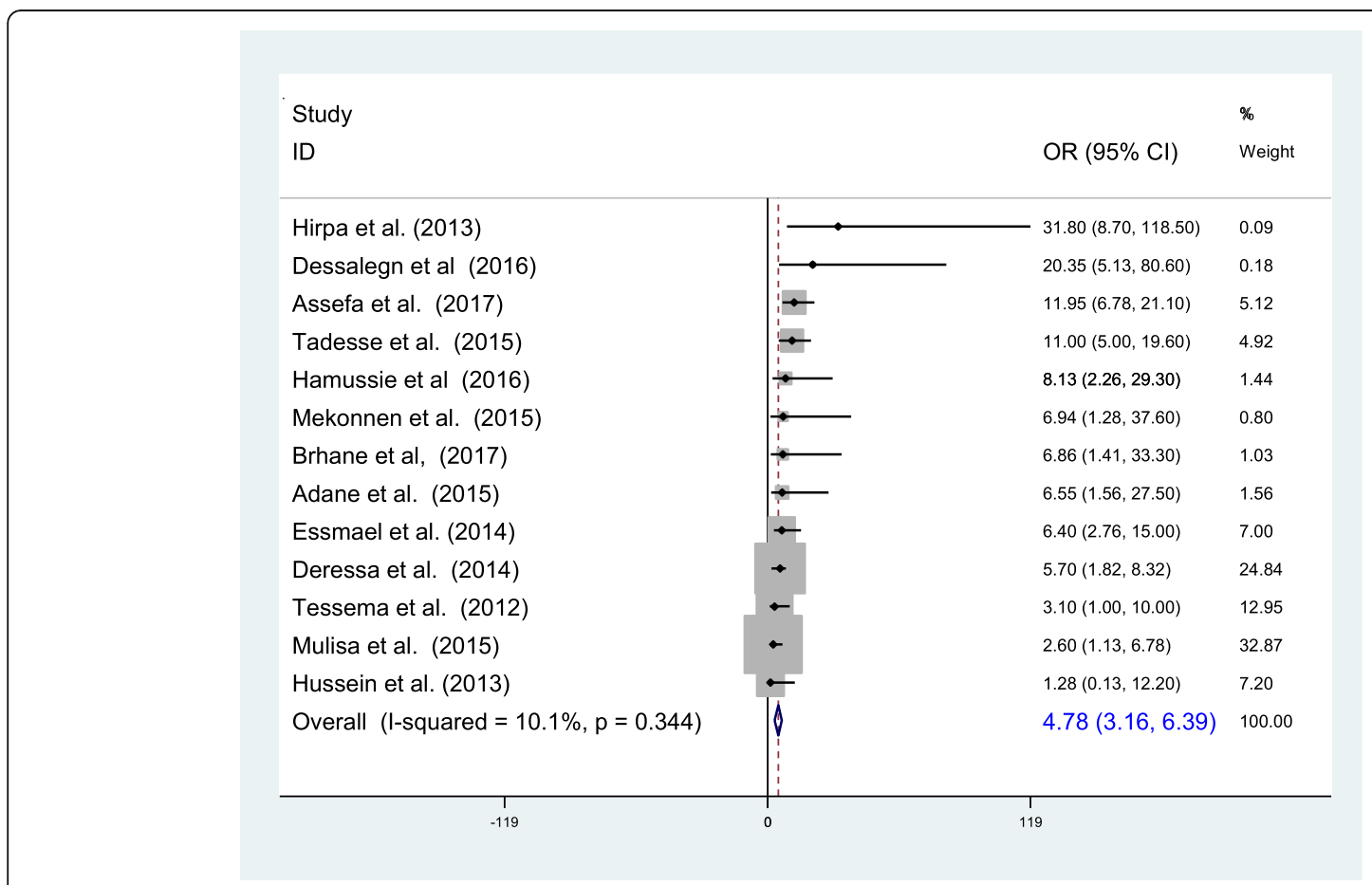

Fig. 5 The pooled odds of previous treatment on MDR-TB prevalence 


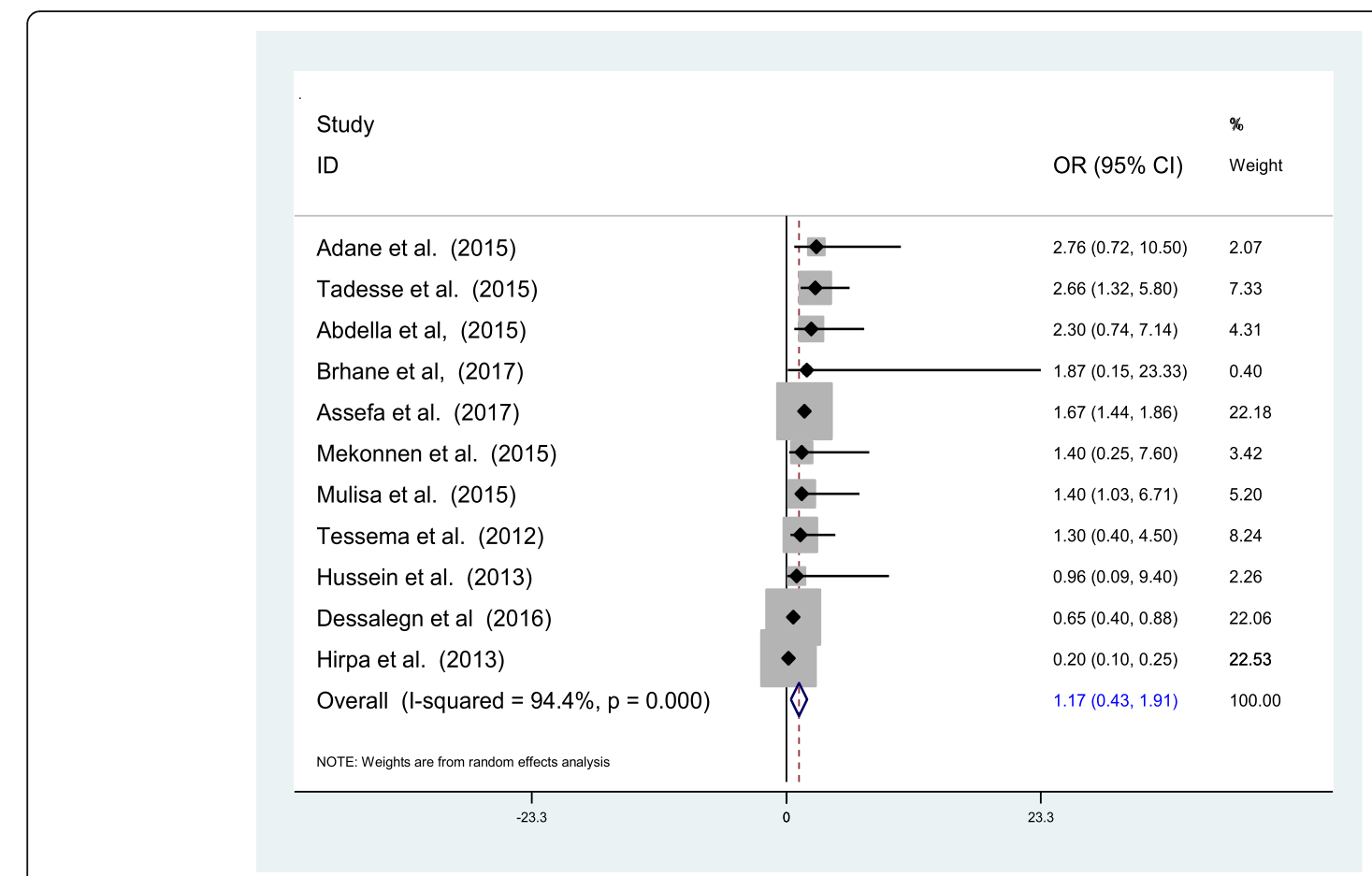

Fig. 6 The pooled odds of HIV infection on MDR-TB prevalence

identified possible determinants and evaluated treatment outcome by reviewing 34 researches conducted in Ethiopia. Within this review 23 researches were pooled together to measure prevalence of MDR-TB and 7.24\% (95\% CI 6.11-8.37) found to have MDR-TB with the rate of $2.18 \%$ (95\% CI $1.44-2.92 \%)$ among new cases and $21.07 \%$ (95\% CI $11.47-30.67 \%$ ) among previously treated patients. History of previous treatment along with some other variables was the major determinants. During the course of treatment $12.25 \%$ (95\% CI 9.39-

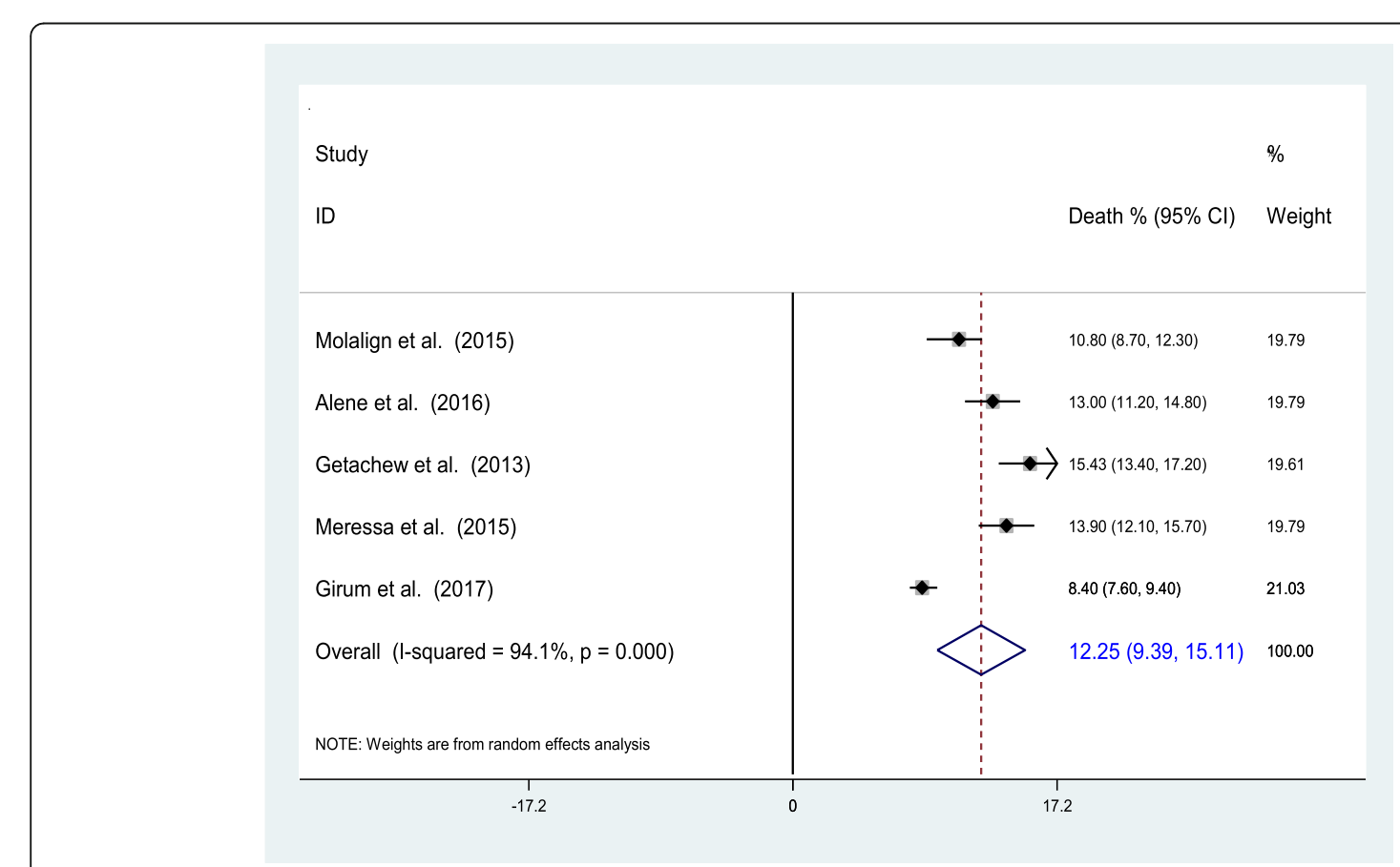

Fig. 7 Forest plot showing the rate of mortality in the course of MDR-TB treatment 
15.11\%) of MDR-TB patients were died mainly associated with complications.

According to this meta-analysis, the pooled prevalence of MDR-TB among newly diagnosed patients was comparable to the finding of previous meta-analysis by Eshetie et al. [50] where $2 \%$ (95\% CI 1-2\%) of newly diagnosed TB patients have MDR-TB. However, it is slightly higher than a previous meta-analysis report that was conducted in Sub-Saharan Africa countries where the prevalence among the new cases was $1.5 \%$ (95\% CI 1.0-2.3) [51] and the national survey report of WHO estimate for Ethiopia $[5,52]$ and the report of national surveys in Benin [53]. On the other hand the prevalence of MDR-TB among new cases was lower than the first Ethiopian national TB survey report of $2.7 \%$ and the survey report of Mozambique and Rwanda [54-56].

The pooled prevalence of MDR-TB among previously treated patients was higher than both of the previously stated researches where 15\% (95\% CI 12 17\%) of patients in Eshetie et al. [50] and 10.3\% (95\% CI 5.8-17.4\%) of patients in Asres et al. [51] have MDR-TB. Likewise, anti-tuberculosis survey in Ethiopia, Benin, Mozambique and Rwanda showed that the prevalence of MDR-TB among previously treated patients was 17.9, 11.1, 11.2 and $9.4 \%$ [53-56] respectively which is by far lower than our pooled estimate.

This discrepancy may be related to the inclusion of many researches conducted in MDR-TB referral centers which knowingly or unknowingly included presumptive MDR-TB cases where the prevalence of MDR-TB is higher. It is logical that patients who have treatment history and currently on treatment are more likely to have MDR-TB than previously treated patient recruited in the community where their TB was treated. There for inclusion of larger samples in hospital based studies particularly in MDR-TB referral centers increases the finding of MDR-TB cases. However in newly diagnosed cases weather they are recruited in the community or in hospitals the prevalence is similar, that is why national surveys and institutional studies have comparable results. In addition to this difference in base line burden, difference in settings and quality of care may have explained the difference [50, 51].

In line to many of the previous reports $[50,51]$ MDR-TB was highly associated to history of previous TB treatment $(\mathrm{OR}=4.78$ (95\% CI 3.16-6.39). The odds of developing MDR-TB among previously treated patients was $8.11(7.52,8.74)$ and 5.69 (3.61-8.96) in Ethiopia [50] and Sub-Saharan countries [51] respectively. As previously treated patients have the chance to acquire a new infection and develop resistance through gene mutation while up on treatment the likely hood of having MDR-TB compared to newly diagnosed TB patient is higher $[5,51]$.
The association between HIV and MDR-TB was not conclusive. Over all pooled odds of HIV was 1.17 (95\% CI 0.43-1.91) which is a positive but insignificant association. Tadesse et al. [44], Mulisa et al. [39] and Assefa et al. [43] reported significantly positive association between HIV infection and MDR-TB. However, a significant positive association between HIV and MDRTB has not been reported from previous reviews in SSA [51] and study results in east Africa and Ethiopia [10, 22, 24, 38]. It is long been known that HIV suppress the human cellular immunity and shorten the period from $\mathrm{TB}$ infection to $\mathrm{TB}$ disease development [8-10]; however, its contribution to the development of drug resistant TB seems to be not significant.

According to the reports of Mulisa et al. [39], Assefa et al. [43] and Brhane et al. [38] exposure to a known MDR-TB case (Contact history) has a significant association with the prevalence of MDR-TB. It is also reported by Federal Ministry of Health of Ethiopia [57] that exposure to a known MDR-TB case along with history of using poor quality $\mathrm{TB}$ drugs and mal-absorption were found to have a positive association with MDR-TB in Ethiopia. Since one of the modes of transmission of MDR-TB is through droplet, a close contact history with a known MDR-TB patient carries higher risk for the development of the disease. Meanwhile, treatment adherence and drug side effect and previous treatment outcome were found to have a positive association with MDR-TB in Ethiopia [41, 48].

Overall, $65-80 \%$ of patients were completed, cured or alive on treatment throughout the period of follow up with in the reviewed researches [45-49] which met the WHO target of 75\% [1] and it is higher than the achievement of most MDR-TB prevalent countries [5, 58]. Also multiples of recent systematic review and meta-analysis reported a lower success rate. Group based meta-analysis Reported pooled treatment success rate of $66.4 \%$ [59], also another individual patient based study review reported a success rate of lower than this fig. [60]. On the other hand some researches from Ethiopia and Haiti reported higher success rates [48, 61].

The proportion of death in our case is also comparable to many other studies [58-60]. However the treatment outcome from a cohort studies may differ depends on whether the research is an open cohort in which outcome may be experienced at the later phase of the treatment or closed cohort in which the outcome is experienced during or before the research is completed. In line to our report a result from a review of 35 articles and another individual patient review reported a death rate of greater than $10 \%[59,60]$.

The treatment advancement through time, patient condition during admission, program organization, the setting and multitude of other factors may contribute 
significantly for difference in success rate. Generally in Ethiopia it was noted from WHO reports and previous studies that the success rate was higher [5]. Most researchers reported that poor outcome of MDR TB treatment is highly contributed by prevalence of HIV co-infection [45-49] and other medical complications. In addition to these, drug side effect and smaller baseline weight were significantly associated with mortality [49]. We have discovered the same relation in which the risk of death in HIV positive individuals was higher than sero-negatives. This is because during HIV infection the prevalence of co-infection, the probability of drug interaction and side effects and overall compliance will decrease which further increase mortality. Similarly, presence of complication contributes for mortality $[47,49]$.

The finding of this review may suffer from the fact that the research is from tuberculosis treatment centers their representativeness for the community is limited. The review was also limited to researches published in English and within the specified data sources as well as it is covering only a specific geographic areas, so that generalization for the country at large may be difficult. Due to institutional based nature of the primary researches inclusion of presumptive cases may be there, which could further increase the prevalent estimate.

\section{Conclusion and recommendation:}

The review showed that MDR-TB is continued to become a serious public health problem in Ethiopia. The prevalence is by far higher than the previous reports among previously treated patients. Meanwhile history of previous treatment along with contact history contributed for the development of the disease. The treatment outcomes reported in this review was comparable with previous studies, yet it is a concern. The main predictors of mortality among MDR-TB patients up on treatment were presence of comorbidities, adverse side effects, mal-absorption, HIV sero-positivity and smaller baseline weight. Thus, proper treatment of drug susceptible TB and early detection and treatment of MDR-TB before complication developed along with prevention of drug side effect and contacts with MDR-TB cases are very important.

\section{Abbreviations \\ DST: Drug Susceptibility Testing; MDR: Multidrug Resistance; MDR- TB: Multidrug-Resistant Tuberculosis; PRISMA: Preferred Reporting Items for Systematic Reviews and Meta-Analyses; PTB: pulmonary Tuberculosis; RR: Rifampicin resistant; TB: Tuberculosis}

\section{Acknowledgments}

All authors who provided us information whenever we contacted them.

\section{Availability of data and materials}

Please contact author for data requests.

\section{Authors' contributions}

TG: Conceived the project, took the primary role in data acquisition, analysis, interpretation writing the manuscript, and publication of the project. All others: revised the project, involved in interpretation and manuscript preparation. Both authors revised the final draft of the manuscript. All authors read and approved the final manuscript.

\section{Authors' information}

TG: is Bsc/public health, MPH in Epidemiology and Biostatistics, Lecturer at Department of Public health, College of Medicine and Health Sciences, Wolkite University, Wolkite, Ethiopia.

\section{Ethics approval and consent to participate}

Not applicable.

\section{Competing interests}

The authors declare that they have no competing interests.

\section{Publisher's Note}

Springer Nature remains neutral with regard to jurisdictional claims in published maps and institutional affiliations.

\section{Author details}

'Department of Public health, college of Medicine and Health Sciences, Wolkite University, Wolkite City, Ethiopia. ${ }^{2}$ Institute of Public health, college of Medicine and Health Sciences, Bahir Dar University, Bahir Dar City, Ethiopia. ${ }^{3}$ Department of Public health, college of Medicine and Health Sciences, Arba Minch University, Arba Minch City, Ethiopia.

Received: 25 January 2018 Accepted: 31 May 2018

Published online: 14 June 2018

\section{References}

1. World health organization. Tuberculosis MDR-TB \& XDR-TB progress report; 2011.

2. WHO. Treatment of tuberculosis: Guidelines For national programs; Geneva; 2003

3. Sharma SK, Mohan A. Multidrug-resistant tuberculosis: a menace that threatens to destabilize tuberculosis control. Chest. 2006;130:261-72.

4. Federal Ministry of Health of Ethiopia (FMOH). Participants' manual: national comprehensive tuberculosis, leprosy and TB/HIV training for general health workers. In: Addis Ababa: FMOH; 2011.

5. World Health Organization. Global tuberculosis report 2017. Geneva, Swizerland: WHO press; 2017.

6. Federal Ministry of Health of Ethiopia (FMOH). Tuberculosis prevention and control programme: special issue for world TB day. 2011;3:17-37.

7. World Health Organization (WHO). Multidrug-resistant tuberculosis (MDR-TB) 2015 update. In: Geneva: WHO; 2015.

8. World Health Organization (WHO): Drug-resistant tuberculosis now at record levels. 2010.

9. World Health Organization (WHO): Anti-tuberculosis drug resistance in the world: fourth global report. Geneva: WHO; 2008. WHO/HTM/TB/2008.394.

10. Migliori GB, Dheda K, Centis R, Mwaba P, Bates M, O'Grady J, et al. Review of multidrug-resistant and extensively drug-resistant TB: global perspectives with a focus on sub-Saharan Africa. Tropical Med Int Health. 2010;15(9): 1052-66.

11. Amor $Y B$, Nemser B, Singh A, Sankin A, Schluger N. Underreported threat of multidrug-resistant tuberculosis in Africa. Emerg Infect Dis. 2008;14(9):1345-52.

12. Sosa AJ, Amábile-Cuevas CF, Byarugaba DK, Hsueh P-R, Kariuki S, Okeke IN Antimicrobial resistance in developing countries. New York: Springer; 2010.

13. Moher D, Liberati A, Tetzlaff J, Altman DG, The PRISMA Group. Preferred reporting items for systematic reviews and metaanalyses: the PRISMA statement. PLoS Med. 2009;6:e1000097.

14. World Health Organization. Definitions and reporting framework for tuberculosis-2013 revision. Geneva: WHO press; 2013.

15. DerSimonian R, Laird N. Meta-analysis in clinical trials. Control Clin Trials. 1986;7:177-88.

16. Demissie M, Gebeyehu M, Berhane Y. Primay resistance to anti-tuberculosis drugs in Addis Ababa. Ethiopia. Int J Tuberc Lung Dis. 1997;1(1):64-7.

17. Gebeyehu M, Lemma E, Eyob G. Prevalence of drug resistance tuberculosis in Arsi zone, Ethiopia. Ethiop J Health Dev. 2001;15:11-6.

18. Bruchfeld J, Aderaye G, Palme IB, Bjorvatn B, Ghebremichael S, Hoffner S, Lindquist L. Molecular epidemiology and drug resistance of Mycobacterium 
tuberculosis isolates from Ethiopian pulmonary tuberculosis patients with and without human immunodeficiency virus infection. J Clin Microbiol. 2002:40(5):1636-43.

19. Asmamaw D, Seyoum B, Makonnen E, Atsebeha H, Woldemeskel D, Yamuah $L$, Addus H, Aseffa A. Primary drug resistance in newly diagnosed smear positive tuberculosis patients in Addis Ababa, Ethiopia. Ethiop MedJ. 2008; 46:367-74

20. Meskel D, Abate G, Lakew M, Goshu S, Aseffa A. Anti-tuberculosis drug resistance among retreatment patients seen at St Peter tuberculosis specialized hospital. Ethiop Med J. 2008;46(3):219-25.

21. Agonafir M, Lemma E, Wolde-Meskel D, Goshu S, Santhanam A. Phenotypic and genotypic analysis of multidrug-resistant tuberculosis in Ethiopia. Int J Tuberc Lung Dis. 2010;14(10):1259-65.

22. Abate $\mathrm{D}$, Taye $\mathrm{B}$, Abisino M, Bidglign S. Epidemiology of anti-tuberculosis drug resistance patterns and trends in tuberculosis referral hospital in Addis Ababa. Ethiopia. BMC Res notes. 2012;5:462.

23. Abebe $G$, Abdissa K, Abdissa A, Apers L, Agonafir M. Relatively low primary drug resistant tuberculosis in southwestern Ethiopia. BMC Res Notes. 2012;5:225.

24. Tessema B, Beer J, Emmrich F, Sack U, Rodloff AC. First and second line antituberculosis drug resistance in Northwest Ethiopia. Int J Tuberc Lung Dis. 2012;16:805-11.

25. Yimer SA, Agonafir M, Derese Y, Sani Y, Bjune GA, HOLM-HANSEN C. Primary drug resistance to anti-tuberculosis drugs in major towns of Amhara region, Ethiopia. APMIS. 2012;120(6):503-9.

26. Hussein B, Debebe T, Wilder-Smith A, Ameni G. Drug susceptibility test on mycobacterium tuberculosis isolated from pulmonary tuberculosis patients in three sites of Ethiopia. A Afr J Microbiol Res. 2013;7(9):791-6.

27. Biadglegne F, Tessema B, Sack U, Rodloff AC. Drug resistance of mycobacterium tuberculosis isolates from tuberculosis lymphadenitis patients in Ethiopia. Indian J Med Res. 2014;140(1):116-22.

28. Esmael A, Ali I, Agonafir M, Endris M, Getahun M, Yarega Z, et al. Drug resistance pattern of mycobacterium tuberculosis in eastern Amhara regional state. Ethiopia J Microb Biochem Technol. 2014;6(2):75-9.

29. Daniel G, Mariam H. Phenotypic and genotypic determination of drug susceptibility and molecular characteri zati on of mycobacteri um tuberculosi s isolates at Debre Berhan referral hospital, Ethiopia. In: Doctoral dissertation, Addis Ababa University. Ethiopia: AAU; 2014. Available at http:// etd.aau.edu.et/handle/123456789/5867.

30. Seyoum B, Demissie M, Worku A, Bekele S, Aseffa A. Prevalence and drug resistance patterns of mycobacterium tuberculosis among new smear positive pulmonary tuberculosis patients in eastern Ethiopia. Tuberc Res Treat. 2014;2014:1-7.

31. Tekle A, Woldeamanuel Y, Asrat D, Beyene D, Aseffa A. Epidemiology and drug resistance pattern of mycobacterium tuberculosis in Northwest Ethiopia: resource limited settings. In: Doctoral dissertation, Addis Ababa University. Ethiopia: AAU; 2014. Available at http://etd.aau.edu.et/handle/ $123456789 / 5791$

32. Abdella K, Abdissa K, Kebede W, Abebe G. Drug resistance patterns of Mycobacterium tuberculosis complex and associated factors among retreatment cases around Jimma. Southwest Ethiopia BMC Public Health. 2015;15(1):599

33. Adane $K$, Ameni $G$, Bekele $S$, Abebe M, Aseffa A. Prevalence and drug resistance profile of mycobacterium tuberculosis isolated from PTB patients attending two public hospitals in east Gojjam zone. northwest Ethiopia BMC Public Health. 2015;15(1):572.

34. Maru M, Mariam SH, Airgecho T, Gadissa E, Aseffa A. Prevalence of tuberculosis, drug susceptibility testing, and genotyping of mycobacterial isolates from pulmonary tuberculosis patients in Dessie, Ethiopia Tuberc Res Treat 2015;2015.

35. Mekonnen F, Tessema B, Moges F, Gelaw A, Eshetie S, Kumera G. Multidrug resistant tuberculosis: prevalence and risk factors in districts of metema and west armachiho. Northwest Ethiopia BMC Infect Dis. 2015;15(1):461.

36. Ali S, Beckert P, Haileamlak A, Weiser A, Pritsch M, Heinrich N. Drug resistance and population structure of M.Tuberculosis isolates from prisons and communities in Ethiopia. BMC Infect Dis. 2016;16:687.

37. Hamusse SD, Teshome D, Hussen MS, Demissie M, Lindtjørn B. Primary and secondary anti-tuberculosis drug resistance in Hitossa District of Arsi zone, Oromia regional state. Central Ethiopia BMC Public Health. 2016;16(1):593.

38. Brhane M, Kebede A, Petros Y. Molecular detection of multidrug-resistant tuberculosis among smear-positive pulmonary tuberculosis patients in Jigjiga town, Ethiopia. Infection and Drug Resistance. 2017;(10):75-83.
39. Mulisa G, Workineh T, Hordofa N, Suaudi M, Abebe G, Jarso G. Multidrugresistant Mycobacterium tuberculosis and associated risk factors in Oromia region of Ethiopia. Int J Infect Dis. 2015;39:57-61.

40. Dessalegn M, Daniel E, Behailu S, Wagnew M, Nyagero J. Predictors of multidrug resistant tuberculosis among adult patients at Saint Peter hospital Addis Ababa. Ethiopia The Pan African Medical Journal. 2016;25(Supp 2):5.

41. Hirpa S, Medhin G, Girma B, Melese M, Mekonen A, Suarez P, et al. Determinants of multidrug-resistant tuberculosis in patients who underwent first-line treatment in Addis Ababa: a case control study. BMC Public Health. 2013;13(1):1-9.

42. Deressa M, Demissie M. Risk factors of multi-drug resistant tuberculosis in Addis Ababa, Ethiopia: a matched case-control study. Open Access Library Journal. 2014;1(3):1-8.

43. Assefa D, Seyoum B, Oljira L. Determinants of multidrug-resistant tuberculosis in Addis Ababa, Ethiopia. Infection and Drug Resistance. 2017;(10):209-13.

44. Tadesse F. Risk factors for multi-drug resistant tuberculosis in Addis Ababa. Ethiopia Universal Journal of Public Health. 2015;3(2):65-70.

45. Molalign S, Wencheko E. Risk factors of mortality in patients with multi-drug resistant TB. Ethiop J Health Dev. 2015;29

46. Alene K, Viney K, Emma S, Tsegaye A, Clements A. Treatment outcomes in patients with multidrug-resistant tuberculosis in north-West Ethiopia. Trop Med Int Health 2016; 00:00

47. Getachew T, Bayray A, Weldearegay B. Survival and predictors of mortality among patients under multi-drug resistant tuberculosis treatment in Ethiopia: St. Peter's specialized tuberculosis hospital, Ethiopia. Int J Pharm Sci Res. 2013:4(2):776-87.

48. Meressa D, Hurtado RM, Andrews JR, et al. Achieving high treatment success for multidrug resistant TB in Africa: initiation and scale-up of MDR TB care in Ethiopia-an observational cohort study. Thorax Online, 2015(207374).

49. Girum T, Tariku Y, Survival Status DS. Treatment outcome of multidrug resistant tuberculosis (MDR-TB) among patients treated in treatment initiation centers (TIC) in South Ethiopia: a retrospective cohort study. Ann Med Health Sci Res. 2017:7:331-6.

50. Eshetie $S$, Gizachew M, Dagnew M, Kumera $G$, Wolde $H$, Ambaw F, et al. Multidrug resistant tuberculosis in Ethiopian settings and its association with previous history of anti-tuberculosis treatment: a systematic review and meta-analysis. BMC Infect Dis. 2017:17:219.

51. Berhan A, Berhan Y, Yizengaw D. A meta-analysis of drug resistant tuberculosis in sub-Saharan Africa: how strongly associated with previous treatment and HIV co-infection? Ethiop J Health Sci. 2013;23(3):271-82.

52. World Health Organization. Antimicrobial resistance global report on surveillance: 2014 summary. Geneva: WHO press; 2014.

53. Affolabi D, Adjagba O, Tanimomo-Kledjo B, Gninafon M, Anagonou S, Portaels F. Anti-tuberculosis drug resistance among new and previously treated pulmonary tuberculosis patients in Cotonou. Benin Int J Tuberc Lung Dis. 2007;11(11):1221-4.

54. Kebede A, Alebachew Z, Tsegaye F, Lemma E, Abebe A, Agonafir M, et al. The first population-based national tuberculosis prevalence survey in Ethiopia, 2010-2011. Int J Tuberc Lung Dis. 2014;18(6):635-9.

55. Gudo PS, Cuna Z, Coelho E, Maungate S, Borroni E, Miotto P, et al. Is multidrug-resistant tuberculosis on the rise in Mozambique? Results of a national drug resistance survey. Eur Respir J. 2011;38(1):222-4.

56. Umubyeyi AN, Vandebriel G, Gasana M, Basinga P, Zawadi J, Gatabazi J, et al. Results of a national survey on drug resistance among pulmonary tuberculosis patients in Rwanda. Int J Tuberc Lung Dis. 2007:11(2):189-94.

57. Federal Ministry of Health of Ethiopia. Guideline for clinical and programmatic management of TB, leprosy and TB/HIV in Ethiopia. 5th ed. Addis Ababa, Ethiopia: $\mathrm{FMOH} ; 2012$.

58. Dennis F, Fuad M, Fraser W, Inés B, Matteo Z, Nguyen L. Etal. Multidrugresistant tuberculosis around the world: what progress has been made? Eur Respir J. 2015:45:150-60.

59. Amal B, Christopher F, Ejaz Q, Razia F, Katherine F, Ernesto J. A systematic review of the effectiveness of Hospitaland ambulatory-based management of multidrug-resistant tuberculosis. Am. J. Trop. Med. Hyg. 2013;89(2):271-80

60. P. Isaakidis, E. C. Casas, M. Das, X. Tseretopoulou, E. E. Ntzani, N. Ford. Treatment outcomes for HIV and MDR-TB co-infected adults and children: systematic review and meta-analysis. INT J TUBERC LUNG DIS2015; 19(8):969-978.

61. Macarthur C, Stalz CV, Serena P, Lauren M, Marie M, Oksana O. Etal. Treatment outcomes for patients with multidrug-resistant tuberculosis in post-earthquake port-au-prince, Haiti. Am J Trop Med Hyg. 2014:91(4):715-21. 